Received 5th December 2016 Accepted 1st February 2017

DOI: $10.1039 / c 6 t a 10450 a$

rsc.li/materials-a

\section{Comparing non-fullerene acceptors with fullerene in polymer solar cells: a case study with FTAZ and PyCNTAZ $\uparrow$}

\author{
Nicole Bauer, ${ }^{a}$ Qianqian Zhang, ${ }^{a}$ Jingbo Zhao, ${ }^{b}$ Long Ye, ${ }^{c}$ Joo-Hyun Kim, ${ }^{c}$ \\ Iordania Constantinou, ${ }^{d}$ Liang Yan, ${ }^{a}$ Franky So, ${ }^{d}$ Harald Ade, ${ }^{c} \mathrm{He}$ Yan $^{\mathrm{b}}$ and Wei You ${ }^{\star a}$
}

Non-fullerene acceptors (NFAs) are becoming a serious contender to fullerene-based electron acceptors in organic photovoltaics, due to their structural versatility and easily tunable optical and electronic properties. However, NFA-based solar cells often have a decreased short-circuit current $\left(J_{\mathrm{SC}}\right)$ and fill factor (FF) compared to their fullerene-based counterparts. Here, we investigate the fundamental causes of this decrease in the performance of solar cells using a non-fullerene acceptor $\left(\mathrm{SF}-\mathrm{PDI}_{2}\right)$ paired with two polymer donors, FTAZ and PyCNTAZ, compared with their fullerene-based counterparts. Through a number of experimental techniques and morphological studies, we show that the SF-PDI 2 -based solar cells suffer from insufficient charge generation, transport, and collection when compared with the PCBM-based solar cells. The SF-PDI 2 -based solar cells show increased bimolecular recombination, which, together with other recombination loss mechanisms in these cells, causes a significant decrease in their $J_{\text {sc }}$ and FF. Notably, the less pure domains, low electron mobility (on the order of $10^{-5} \mathrm{~cm}^{2} \mathrm{~V}^{-1}$ $\mathrm{s}^{-1}$ ), and imbalanced mobility (in regard to the hole mobility) further explain the low FF. On the other hand, the higher open-circuit voltage $\left(V_{\text {oc }}\right)$ in the SF-PDI 2 devices is mainly due to the increase in the CT state energy. It is worth mentioning that the PyCNTAZ-based devices show an ultralow charge separation energy $\left(\Delta E_{C S}\right)$, close to $0 \mathrm{eV}$. Our results demonstrate that further increasing the mobility (both of electrons and holes) in these NFA-based solar cells would be a viable approach to further enhance the efficiency of these new types of solar cells, ideally, without losing the high $V_{\text {oc }}$ of such cells.

\section{Introduction}

Organic photovoltaics (OPVs) are promising candidates for solar energy applications for reasons including the potentially low fabrication cost (from both a processing and a materials' perspective), light weight, and mechanical flexibility of the devices when compared to silicon solar cells. The most common electron acceptors used in OPVs are fullerene derivatives because of their high electron affinity and relatively high electron mobility. ${ }^{\mathbf{1}}$ Despite these advantages, fullerenes have a number of drawbacks, including difficult synthesis processes, rather fixed energy levels, and poor light absorption in the visible region. To address these

${ }^{a}$ Department of Chemistry, University of North Carolina at Chapel Hill, Chapel Hill, North Carolina 27599, USA. E-mail: wyou@unc.edu

${ }^{b}$ Department of Chemistry, Hong Kong Branch of Chinese National Engineering Research Center for Tissue Restoration \& Reconstruction, Hong Kong University of Science and Technology, Clear Water Bay, Kowloon, Hong Kong

'Department of Physics and ORaCEL, North Carolina State University, Raleigh, North Carolina 27695, USA

${ }^{d}$ Department of Materials Science and Engineering; North Carolina State University, Raleigh, North Carolina 27606, USA

$\dagger$ Electronic supplementary information (ESI) available. See DOI: $10.1039 /$ c6ta10450a fullerene-associated issues, non-fullerene acceptors (NFAs) have gained significant momentum in recent years, due to their many advantages over traditional fullerenes. ${ }^{2}$ For example, one can easily tune the optical and electronic properties of NFAs via molecular designing, which could allow for enhanced open-circuit voltage $\left(V_{\mathrm{oc}}\right)$ from their energy levels matching those of the donor polymer ${ }^{3,4}$ and increased current $\left(J_{\mathrm{sc}}\right)$ from their complementary absorption to that of the donor polymer. ${ }^{5,6}$ Benefitting from the experiences gained and lessons learned from decades of research on fullerene-based bulk heterojunction (BHJ) systems, the community has made rapid progress in the past two years with record high efficiency reaching $\sim 12 \%$ for NFA-based devices, ${ }^{7,8}$ on par with the highest efficiency achieved for fullerene blends. ${ }^{9-11}$

Though a higher $V_{\text {oc }}$ is usually obtained for NFA-based organic solar cells than that of their fullerene-based counterparts, the NFA-based devices often suffer from a noticeably reduced shortcircuit current $\left(J_{\mathrm{sc}}\right)$ and/or fill factor $(\mathrm{FF}) .{ }^{12}$ Since both $J_{\mathrm{sc}}$ and FF are closely related to the loss mechanisms in photovoltaic devices (e.g., bimolecular recombination), ${ }^{\mathbf{1 3 , 1 4}}$ it is important to understand such loss mechanisms in NFA-based solar cells, ideally in a comparative manner with the corresponding solar cells based on fullerenes. Unfortunately, such studies have only begun to emerge. ${ }^{15,16}$ 

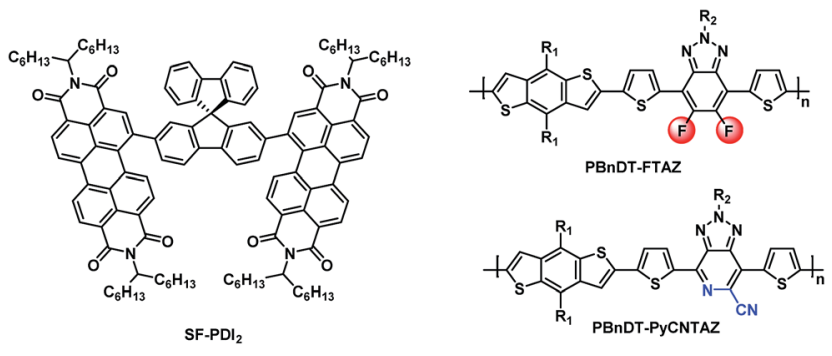

Chart 1 Chemical structures of SF-PDI 2, FTAZ, and PyCNTAZ.

We set our study by first choosing SF-PDI ${ }_{2}$ as the small molecule, non-fullerene electron acceptor. In a previous study by Zhao et al., SF-PDI ${ }_{2}$ was paired with the polymer donor PffBT4T-2DT and achieved a high $V_{\text {oc }}$ of $0.98 \mathrm{~V}$ in their BHJ devices, leading to a respectable power conversion efficiency (PCE) of $6.3 \% .{ }^{17}$ We then selected two TAZ-based donor polymers, FTAZ and PyCNTAZ (structures given in Chart 1), that have shown impressive device performance in their $\mathrm{BHJ}$ solar cells based on fullerene acceptors. ${ }^{18,19}$ The first donor polymer, FTAZ, containing a fluorinated benzotriazole as the electronaccepting moiety, was reported by Price et al. in 2011. ${ }^{18}$ They obtained a relatively high $V_{\text {oc }}$ of $0.79 \mathrm{~V}$ with an impressive $\mathrm{FF}$ of $72 \%$, giving an overall PCE of over $7 \% .{ }^{14,20}$ The other donor polymer chosen for this work, PyCNTAZ, was introduced by Li et al. in 2015. ${ }^{19}$ In BHJ solar cells with PCBM, PyCNTAZ displayed a $V_{\text {oc }}$ of $0.96 \mathrm{~V}$, higher than that of FTAZ, leading to a higher PCE of $8.37 \%$ at an optimized active layer thickness of $300 \mathrm{~nm}$.

In this study, we aim to directly compare the photovoltaic performance of four $\mathrm{BHJ}$ blends based on two acceptors (SF$\mathrm{PDI}_{2}$ and $\mathrm{PC}_{61} \mathrm{BM}$, referred to here as PCBM) and two donor polymers (FTAZ and PyCNTAZ), and investigate the device physics and morphology to determine the origins of the differences in performance. Notably, for both polymers, the SF$\mathrm{PDI}_{2}$-based photovoltaic device has a higher $V_{\text {oc }}$ than the PCBMbased counterpart. This higher $V_{\text {oc }}$ is directly correlated with the higher charge transfer state energy $\left(E_{\mathrm{CT}}\right)$ of the SF-PDI ${ }_{2}$ blend, mainly due to the higher-lying lowest unoccupied molecular orbital (LUMO) level of SF-PDI 2 . However, the SF-PDI 2 -based device displays a lower FF than the PCBM-based device due to a mobility imbalance and less pure domains. Furthermore, the SF-PDI ${ }_{2}$-based device shows a lower $J_{\text {sc }}$, which can be ascribed to inefficient charge transfer from the donor polymer to the nonfullerene acceptor (i.e., SF-PDI ${ }_{2}$ ) and increased non-geminate recombination in such non-fullerene acceptor-based blends.

\section{Results and discussion}

\section{Photovoltaic performance}

To investigate the performance differences displayed between devices containing a fullerene and a non-fullerene acceptor, the small molecule acceptor SF-PDI ${ }_{2}$ was paired with the donor polymers FTAZ and PyCNTAZ in bulk heterojunction (BHJ) solar cells. Devices were also fabricated using PCBM as the acceptor with both polymers for comparison. A conventional device
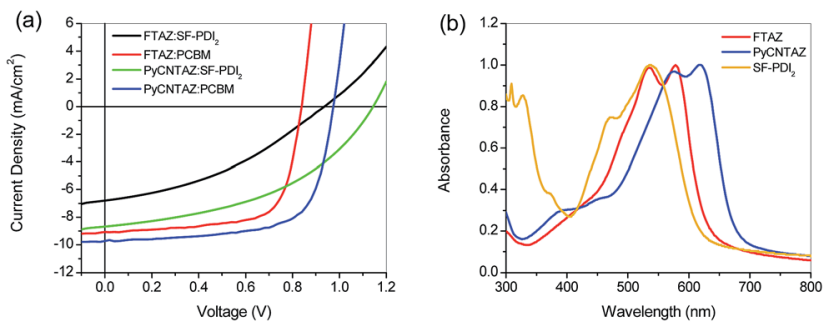

Fig. 1 (a) J-V characteristics of SF-PDI - and PCBM-based solar cells; (b) normalized absorption spectra of neat FTAZ, PyCNTAZ and SF-PDI 2 films.

Table 1 Photovoltaic characteristics of SF-PDI $2^{-}$and PCBM-based solar cells

\begin{tabular}{|c|c|c|c|c|}
\hline Blend & $\begin{array}{l}J_{\mathrm{sc}} \\
\left(\mathrm{mA} \mathrm{cm}^{-2}\right)\end{array}$ & $V_{\mathrm{oc}}(\mathrm{V})$ & $\mathrm{FF}(\%)$ & PCE (\%) \\
\hline FTAZ:SF-PDI ${ }_{2}$ & $6.70 \pm 0.29$ & $0.935 \pm 0.005$ & $36.7 \pm 1.6$ & $2.30 \pm 0.15$ \\
\hline FTAZ:PCBM & $9.16 \pm 0.32$ & $0.846 \pm 0.007$ & $73.3 \pm 2.0$ & $5.68 \pm 0.23$ \\
\hline PyCNTAZ:SF-PDI ${ }_{2}$ & $8.15 \pm 0.12$ & $1.152 \pm 0.003$ & $46.5 \pm 1.3$ & $4.37 \pm 0.17$ \\
\hline PyCNTAZ:PCBM & $10.10 \pm 0.38$ & $0.975 \pm 0.003$ & $64.8 \pm 2.3$ & $6.39 \pm 0.41$ \\
\hline
\end{tabular}

configuration was used (ITO/HTL/active layer/Ca/Al), where the hole transport layer (HTL) was poly(3,4-ethylenedioxythiophene)-poly(styrenesulfonate) (PEDOT:PSS) for FTAZ-based devices and copper thiocyanate (CuSCN) for PyCNTAZ-based devices. Due to the deeper HOMO (highest occupied molecular orbital) level of PyCNTAZ, using CuSCN as the HTL can help improve the photovoltaic device performance when compared to PEDOT:PSS as the HTL, as we previously demonstrated..$^{19}$ Active layer thicknesses for all four devices were kept at $\sim 150 \mathrm{~nm}$ to minimize thickness effects on performance, and the donor : acceptor (D : A) ratio in the BHJ blend for all devices was $1: 2$ by weight. The $J-V$ curves are shown in Fig. 1 a and the photovoltaic characteristics are summarized in Table 1 . For the SF-PDI ${ }_{2}$ blends, the PyCNTAZ-based device displays a higher $J_{\mathrm{sc}}$ than the FTAZ-based device, which can be ascribed to less overlapping/more complementary absorption of PyCNTAZ and SF-PDI ${ }_{2}$ (Fig. 1b) and efficient utilization of both components to generate current. Pleasingly, when compared to the fullerenebased device, the SF-PDI ${ }_{2}$-based device gives a higher $V_{\mathrm{oc}}$ value for both polymers than the PCBM-based one, i.e., $0.935 \mathrm{~V}$ vs. $0.846 \mathrm{~V}$ for FTAZ:SF-PDI ${ }_{2}$ and FTAZ:PCBM, respectively, and $1.152 \mathrm{~V}$ vs. $0.975 \mathrm{~V}$ for PyCNTAZ:SF-PDI ${ }_{2}$ and PyCNTAZ:PCBM, respectively. However, the $\mathrm{SF}-\mathrm{PDI}_{2}$-based device has a lower $J_{\mathrm{sc}}$ and FF than the PCBM-based device, leading to a lower power conversion efficiency (PCE) by the former device for both polymers. Below, we will discuss each device characteristic in further detail and strive to identify the underlying reasons for the observed trends.

\section{Open circuit voltage $\left(V_{o c}\right)$}

To determine the cause of the higher $V_{\mathrm{oc}}$ for SF-PDI ${ }_{2}$-based devices, we first considered the energy levels of the donors 


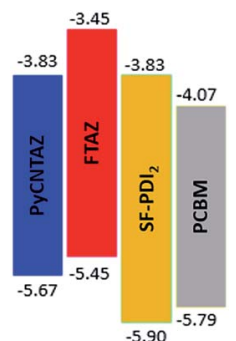

Fig. 2 HOMO and LUMO levels of PyCNTAZ, FTAZ, SF-PDI 2 , and PCBM.

and acceptors. Such values can be extracted from previous reports, ${ }^{17-19,21}$ which are presented in Fig. 2. It has long been argued that $V_{\mathrm{oc}}$ is primarily proportional to the difference between the LUMO energy level of the acceptor and the HOMO energy level of the donor, if ohmic contacts are achieved at both cathode and anode interfaces. SF-PDI ${ }_{2}$ has a higher-lying LUMO level than PCBM, $-3.83 \mathrm{eV}$ compared to $-4.07 \mathrm{eV}$, respectively. This difference $(\sim 0.2 \mathrm{eV})$ certainly accounts for a higher $V_{\text {oc }}$ for both SF-PDI ${ }_{2}$-based solar cells; yet the observed $V_{\text {oc }}$ difference $(\sim 0.09 \mathrm{~V}$ for FTAZ-based devices and $\sim 0.15 \mathrm{~V}$ for PyCNTAZ-based devices) needs further investigation (vide infra).

Vandewal et al. demonstrated that $V_{\mathrm{oc}}$ is primarily determined by the interfacial charge-transfer (CT) states between the donor and the acceptor, with additional loss coming from the radiative emission and non-radiative emission. ${ }^{22,23}$ Eqn (1) presents the relationship,

$$
\begin{aligned}
V_{\mathrm{oc}} & =\frac{k T}{q} \ln \left(\frac{J_{\mathrm{ph}}}{J_{0}}+1\right) \\
& =\frac{E_{\mathrm{CT}}}{q}+\frac{k T}{q} \ln \left(\frac{J_{\mathrm{sc}} h^{3} c^{2}}{f q 2 \pi\left(E_{\mathrm{CT}}-\lambda\right)}\right)+\frac{k T}{q} \ln \left(\mathrm{EQE}_{\mathrm{EL}}\right)
\end{aligned}
$$

which can also be re-written as ( $q$ is the elementary charge, i.e., e)

$$
e V_{\mathrm{oc}}=E_{\mathrm{CT}}+k T \ln \left(\frac{J_{\mathrm{sc}} h^{3} c^{2}}{f q 2 \pi\left(E_{\mathrm{CT}}-\lambda\right)}\right)+k T \ln \left(\mathrm{EQE}_{\mathrm{EL}}\right)
$$

According to Faist et al.,${ }^{24}$ the energy difference between $e V_{\mathrm{oc}}$ and $E_{\mathrm{CT}}$ is the loss due to the non-geminate recombination (i.e., $\left.\Delta E_{\mathrm{NG}}=E_{\mathrm{CT}}-e V_{\mathrm{oc}}\right)$, and the charge separation energy/exciton splitting energy $\left(\Delta E_{\mathrm{CS}}=E_{\mathrm{opt}}-E_{\mathrm{CT}}\right)$ is the difference between
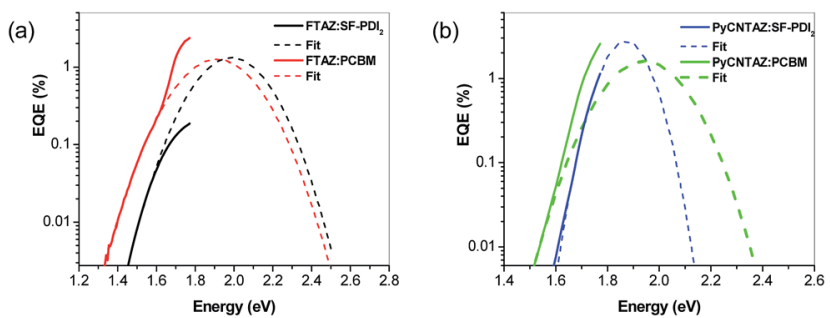

Fig. 3 Measured low energy external quantum efficiency (EQE) curves and calculated fittings of (a) FTAZ and (b) PyCNTAZ based solar cells. the optical gap $\left(E_{\mathrm{opt}}\right)$ and the energy of the CT state manifold. Unlike a typical inorganic solar cell where there are essentially no CT states (thus $\Delta E_{\mathrm{CS}} \sim 0$ ), the presence of the CT state manifold constructs a significant channel of energy loss $\left(\Delta E_{\mathrm{CS}}\right)$, manifested by the typically observed smaller $V_{\text {oc }}$ when compared with the band gap (i.e., $e V_{\mathrm{oc}}=E_{\mathrm{opt}}-\Delta E_{\mathrm{CS}}-\Delta E_{\mathrm{NG}}$ ). Experimentally, high sensitivity external quantum efficiency (EQE) measurements were performed and the resulting spectra (Fig. 3) were fitted to obtain an estimate for $E_{\mathrm{CT}}$ (Table 2) using eqn (3). ${ }^{21}$

$$
\left.\mathrm{EQE}(E) \propto \frac{1}{E \sqrt{4 \pi \lambda k T}} \exp -\frac{\left(E_{\mathrm{CT}}+\lambda-E\right)^{2}}{4 \lambda k T}\right)
$$

With the optically determined band gap $\left(E_{\text {opt }}\right)$ and measured $V_{\text {oc }}$, the aforementioned $\Delta E_{\mathrm{CS}}$ and $\Delta E_{\mathrm{NG}}$ can thereby be determined for each blend (Table 2).

For both polymers, the difference in $E_{\mathrm{CT}}$ between the fullerene and the non-fullerene device is almost identical to the difference in $V_{\text {oc }}$ (numerically), for example, a $\Delta E_{\text {Cт }}$ of $0.15 \mathrm{eV}$ (1.55-1.39) vs. a $\Delta V_{\text {oc }}$ of $0.16(0.93-0.77)$ for FTAZ. This observation implies that the $V_{\mathrm{oc}}$ loss in these systems is independent of the choice of acceptor (i.e., SF-PDI 2 or PCBM). Furthermore, $\Delta E_{\mathrm{NG}}$ is similar for all devices at $\sim 0.6 \mathrm{eV}$, indicating that $V_{\mathrm{oc}}$ losses from non-geminate recombination are also very similar in all four blends. However, while the charge separation energy $\left(\Delta E_{\mathrm{CS}}\right)$ for FTAZ-based cells is 'normal', $0.27 \mathrm{eV}$ for FTAZ:PCBM and $0.44 \mathrm{eV}$ for FTAZ:SF-PDI ${ }_{2}$, the PyCNTAZ-based devices display an exceptionally low driving force $\left(\Delta E_{\mathrm{CS}}\right)$ of $\sim 0.03 \mathrm{eV}$ for both devices. While not as common, devices that maintain efficient charge separation and high performance but display a low $\Delta E_{\mathrm{CS}}$ have been recently reported, interestingly, also for SF-PDI ${ }_{2}$-based devices. ${ }^{25}$ This exciting discovery certainly warrants further investigation, since achieving a low $\Delta E_{\mathrm{CS}}$ is a very promising approach to further enhance the efficiency of polymer solar cells.

\section{Short circuit current $\left(J_{s c}\right)$}

Comparing the $J_{\mathrm{sc}}$ values in Table 1 raises one important question we try to answer with this study: why is $J_{\mathrm{sc}}$ lower in both cases for SF-PDI ${ }_{2}$-based devices when compared with PCBM-based devices? In fact, there are multiple possible causes which can occur at various stages of the photovoltaic process (i.e., charge generation, charge transport and charge collection), to account for a lowered $J_{\mathrm{sc}}$. For example, insufficient exciton quenching could lead to insufficient charge generation, and bimolecular recombination could compete with charge transport to the electrode, to name a few.

We first measured the photoluminescence (PL) quenching to study exciton splitting/charge generation in the BHJ blends, and the data are presented in Fig. 4. Due to the absorption overlap of SF-PDI ${ }_{2}$ with the two donor polymers, we chose to use $\mathrm{D}: \mathrm{A}=$ $10: 1$ to allow for quantitative determination of PL quenching (PL quenching with D: A = 1: 2 in Fig. $\mathrm{S} 3 \dagger$ ). It is clear from the results that $\mathrm{SF}_{-} \mathrm{PDI}_{2}$-based blends show less PL quenching than PCBM-based blends (for both FTAZ and PyCNTAZ), indicating 
Table $2 V_{\mathrm{OC}}, E_{\mathrm{CT}}$, and energy losses of FTAZ- and PyCNTAZ-based solar cells

\begin{tabular}{|c|c|c|c|c|c|c|}
\hline Blend & $V_{\mathrm{oc}}^{b}(\mathrm{~V})$ & $E_{\text {opt }}(\mathrm{eV})$ & $E_{\mathrm{CT}}(\mathrm{eV})$ & $\begin{array}{l}\Delta E_{\mathrm{loss}}(\mathrm{eV}) \\
E_{\mathrm{opt}}-e V_{\mathrm{oc}}\end{array}$ & $\begin{array}{l}\Delta E_{\mathrm{CS}}(\mathrm{eV}) \\
E_{\mathrm{opt}}-E_{\mathrm{CT}}\end{array}$ & $\begin{array}{l}\Delta E_{\mathrm{NG}}(\mathrm{eV}) \\
E_{\mathrm{CT}}-e V_{\mathrm{oc}}\end{array}$ \\
\hline FTAZ:SF-PDI ${ }_{2}$ & 0.93 & 1.99 & 1.55 & 1.06 & 0.44 & 0.62 \\
\hline PyCNTAZ:SF-PDI ${ }_{2}$ & 1.10 & 1.80 & 1.76 & 0.70 & 0.04 & 0.66 \\
\hline PyCNTAZ:PCBM & 0.97 & $1.66^{a}$ & 1.63 & 0.69 & 0.03 & 0.66 \\
\hline
\end{tabular}

${ }^{a}$ Please note that the band gap of PCBM was used here as the band gap of the polymer is larger than the band gap of PCBM. ${ }^{b}$ The top contacts for these devices ( $\mathrm{LiF} / \mathrm{Al})$ were evaporated at NCSU after spin casting the active layer at UNC.

that there may be an issue with donor to acceptor charge transfer in the SF-PDI 2 -based systems, especially for PyCNTAZ. Incomplete charge transfer in the device would decrease the $J_{\mathrm{sc}}$ at the source of charge generation. For the FTAZ-based blends (Fig. 4a), the amount of quenching was $82 \%$ and $96 \%$ for FTAZ:SF-PDI ${ }_{2}$ and FTAZ:PCBM, respectively, suggesting that the driving force $\left(\Delta E_{\mathrm{CS}}>0.2 \mathrm{eV}\right)$ is large enough to allow only $10 \%$ acceptor to sufficiently quench the PL of FTAZ with either SF$\mathrm{PDI}_{2}$ or PCBM. On the other hand, for PyCNTAZ-based blends (Fig. 4b), the amount of quenching was only $34 \%$ and $81 \%$ for PyCNTAZ:SF-PDI ${ }_{2}$ and PyCNTAZ:PCBM, respectively. It is possible that due to the low driving force $\left(\Delta E_{\mathrm{CS}} \sim 0 \mathrm{eV}\right)$ of the PyCNTAZ blends, energy transfer may also play an important role in the PL quenching, in addition to photo-induced charge transfer. In the PyCNTAZ:PCBM blend, though the driving force is small $\left(\Delta E_{\mathrm{CS}} \sim 0 \mathrm{eV}\right), \mathrm{PCBM}$ has a smaller band gap and there can be both energy transfer and charge transfer in the PyCNTAZ:PCBM system. Thus, $\sim 10 \%$ PCBM is still able to quench a much larger degree of the PL of PyCNTAZ (Fig. 4b). However, in the PyCNTAZ:SF-PDI ${ }_{2}$ blend, there is essentially no energy transfer for quenching the PL of PyCNTAZ because the band gap of $\mathrm{SF}_{-\mathrm{PDI}}$ is larger than that of PyCNTAZ; thus, the PL quenching is purely dependent upon the photo-induced charge transfer for the PyCNTAZ:SF-PDI 2 blend, which is not sufficient due to the low driving force $\left(\Delta E_{\mathrm{CS}} \sim 0 \mathrm{eV}\right)$.

We next attempted to probe the recombination mechanisms occurring in these devices, since the recombination, in particular the bimolecular recombination, is known to have a strong impact on charge transport. To this end, light intensity dependence of $J_{\mathrm{sc}}$ and $V_{\mathrm{oc}}$ was measured for the four devices. On the one hand, $J_{\mathrm{sc}}$ is known to have a power law dependence on light intensity, following the relationship
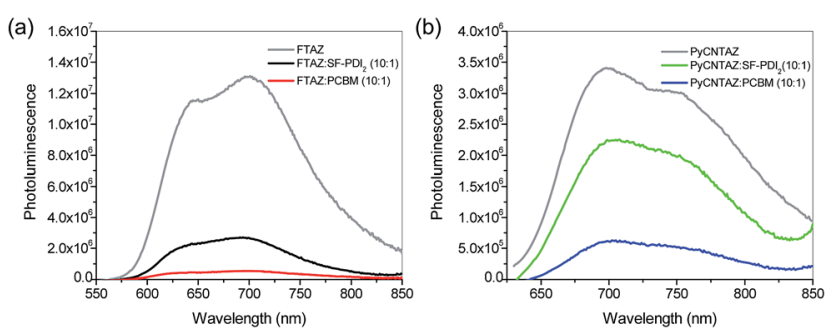

Fig. 4 Photoluminescence of neat (a) FTAZ (excitation at $532 \mathrm{~nm}$ ) and (b) PyCNTAZ (excitation at $618 \mathrm{~nm}$ ) films and the blend films with SF$\mathrm{PDI}_{2}$ and PCBM (D:A = $\left.10: 1\right)$.

$$
J_{\mathrm{sc}} \propto I^{\alpha}
$$

where $I$ is the light intensity and $\alpha$ is the slope of the log-log plot of $J_{\mathrm{sc}} v s$. intensity. ${ }^{26}$ An $\alpha$ value close to unity is indicative of weak bimolecular recombination under short-circuit conditions. The log plot of $J_{\mathrm{sc}} v s$. light intensity is displayed in Fig. 5a. For both polymers, the $\alpha$ value for the fullerene-based device is closer to one than that of the non-fullerene device, suggesting increased bimolecular recombination in the SF-PDI 2 -based devices at short circuit. The increased recombination would compete with charge extraction and lead to a lower current in the non-fullerene based devices.

On the other hand, when bimolecular recombination is the only loss mechanism, $V_{\text {oc }}$ can be related to light intensity by the following equation:

$$
V_{\mathrm{oc}}=\frac{E_{\mathrm{gap}}}{q}-\frac{k T}{q} \ln \left[\frac{\left(1-P_{\mathrm{D}}\right) \gamma N_{\mathrm{c}}{ }^{2}}{P_{\mathrm{D}} G}\right]
$$

where $E_{\text {gap }}$ is the $\mathrm{HOMO}_{\text {donor }}-\mathrm{LUMO}_{\text {acceptor }}$ difference, $k$ is the Boltzmann constant, $T$ is the temperature in Kelvin, $q$ is the elementary charge (i.e., $e$ ), $P_{\mathrm{D}}$ is the dissociation probability of electron-hole pairs, $\gamma$ is the recombination constant, $N_{\mathrm{C}}$ is the effective density of states, and $G$ is the generation rate of bound electron-hole pairs. ${ }^{26}$ In this equation, $G$ is proportional to the light intensity, and a semi-log plot of $V_{\mathrm{oc}} v s$. light intensity will yield a slope of $k T / q$ if bimolecular recombination is the sole loss mechanism. Fig. 5b presents the $V_{\mathrm{oc}} v s$. light intensity data for all four devices. For both polymers, the PCBM-based blends have a slope very close to $k T / q$, indicating that bimolecular recombination is the major loss mechanism under open-circuit conditions in these devices. However, the FTAZ:SF-PDI ${ }_{2}$ device shows a slope greater than $k T / q$ whereas PyCNTAZ:SF-PDI ${ }_{2}$ has a slope less than $k T / q$. These results indicate that the FTAZ:SF-
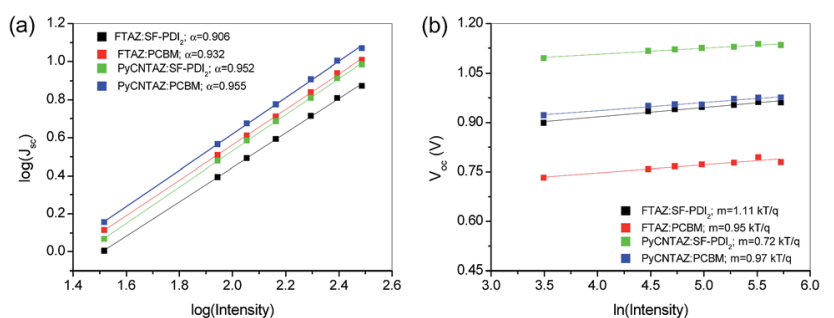

Fig. 5 Light intensity dependence of (a) short-circuit current and (b) open-circuit voltage. 
$\mathrm{PDI}_{2}$ device suffers from a combination of trap-assisted and bimolecular recombination under open-circuit conditions, ${ }^{27,28}$ while PyCNTAZ:SF-PDI ${ }_{2}$ is mainly affected by surface recombination. ${ }^{29,30}$ Due to these additional recombination channels, the number of charges that can successfully traverse the active layer and make it to the electrode will be less for the SF-PDI 2 -based devices compared to the PCBM-based ones, which would decrease the current.

Finally, we studied the charge collection by comparing the charge collection probability, $P(E, T)$, for all blends. Experimentally, the photocurrent density $\left(J_{\mathrm{ph}}\right)$ was first measured as a function of the effective voltage $V_{\text {eff }}$ (Fig. 6a) for each device. $J_{\mathrm{ph}}$ is defined as $J_{\mathrm{L}}-J_{\mathrm{D}}$, where $J_{\mathrm{L}}$ is the current density under illumination and $J_{\mathrm{D}}$ is the current density in the dark, and $V_{\text {eff }}$ is $V_{0}-V$, where $V_{0}$ is the voltage at which $J_{\mathrm{ph}}=0 .{ }^{31,32}$ The photocurrent density $\left(J_{\mathrm{ph}}\right)$ was then used to calculate the charge collection probability, $P(E, T)$, for each blend using the equation

$$
P(E, T)=\frac{J_{\mathrm{ph}}}{J_{\mathrm{ph}, \mathrm{sat}}}
$$

where $J_{\mathrm{ph} \text {,sat }}$ is the saturation photocurrent density of the device. $^{32}$ Under short-circuit conditions, the PCBM-based devices have a higher $P(E, T)$ than the non-fullerene devices, 92.4\% compared to $73.5 \%$ for FTAZ:PCBM and FTAZ:SF-PDI respectively, and $89.3 \%$ compared to $79.8 \%$ for PyCNTAZ:PCBM and PyCNTAZ:SF-PDI ${ }_{2}$, respectively. These results indicate that the charge collection process is more efficient in the fullerene devices, which contributes to the higher $J_{\mathrm{sc}}$ of the fullerenebased devices for both polymers.

\section{Fill factor (FF)}

We previously showed that the main reason for the unusually high FF of the FTAZ:PCBM device was the high hole mobility $\left(1.2 \times 10^{-3} \mathrm{~cm}^{2} \mathrm{~V}^{-1} \mathrm{~s}^{-1}\right)$ and the balanced mobility (i.e., electron mobility on the same order of magnitude, $\sim 5 \times 10^{-3} \mathrm{~cm}^{2}$ $\left.\mathrm{V}^{-1} \mathrm{~s}^{-1}\right) \cdot{ }^{14}$ Indeed, the space-charge limited current (SCLC) mobilities of the PCBM-based devices in this study further confirmed the balanced mobilities (Table 3) for both polymers.

However, when switching out PCBM for SF-PDI 2 , the SF-PDI based devices show a large imbalance in the electron and the hole mobility for both polymers (Table 3). Proctor et al. have previously shown that low and imbalanced mobilities can reduce the fill factor of a solar cell. ${ }^{33}$ In our case, the hole mobility of the non-fullerene devices is one order of magnitude
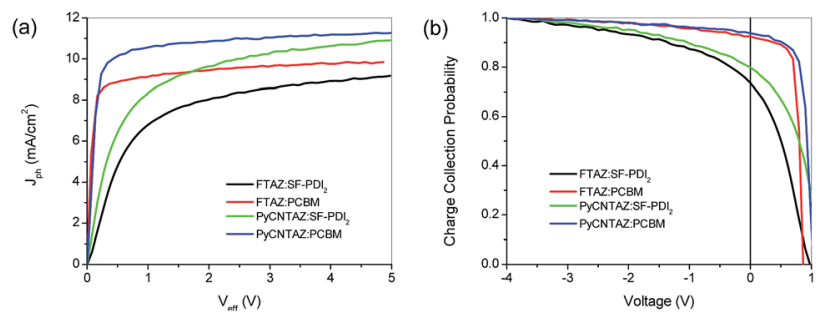

Fig. 6 (a) Photocurrent density and (b) charge collection probability $(P(E, T))$ of SF-PDI $2^{-}$and PCBM-based solar cells.
Table 3 Electron and hole mobilities of SF-PDI $2^{-}$and PCBM-based solar cells

\begin{tabular}{lcc}
\hline Blend & $\begin{array}{l}\text { Electron mobility } \\
\left(\times 10^{-3} \mathrm{~cm}^{2} \mathrm{~V}^{-1} \mathrm{~s}^{-1}\right)\end{array}$ & $\begin{array}{l}\text { Hole mobility } \\
\left(\times 10^{-3} \mathrm{~cm}^{2} \mathrm{~V}^{-1} \mathrm{~s}^{-1}\right)\end{array}$ \\
\hline FTAZ:SF-PDI $_{2}$ & $0.0368 \pm 0.007$ & $0.635 \pm 0.219$ \\
FTAZ:PCBM & $1.68 \pm 0.565$ & $4.23 \pm 3.33$ \\
PyCNTAZ:SF-PDI $_{2}$ & $0.019 \pm 0.003$ & $0.504 \pm 0.136$ \\
PyCNTAZ:PCBM & $2.28 \pm 1.43$ & $1.26 \pm 0.515$
\end{tabular}

larger than the electron mobility; this large mobility imbalance is likely a major cause of the decreased $\mathrm{FF}$ in the SF-PDI 2 -based devices.

In addition to the mobility imbalance, the $\mathrm{SF}-\mathrm{PDI}_{2}$-based devices also display lower mobilities overall, in particular, for electrons (on the order of $10^{-5} \mathrm{~cm}^{2} \mathrm{~V}^{-1} \mathrm{~s}^{-1}$ ). This may be due to the morphology of the SF-PDI ${ }_{2}$-based films, which will be discussed in more detail in the next section. The low, imbalanced mobilities can lead to a build-up of space charge and exacerbate the charge transport via increased bimolecular recombination. This agrees with the results of the light intensity measurements, which indicate that the SF-PDI ${ }_{2}$-based devices suffer from more recombination than the PCBM-based devices. The increased recombination would not only decrease the $J_{\mathrm{sc}}$ as mentioned previously, but also have a negative effect on the FF for the nonfullerene based devices.

\section{Morphology}

The morphology of $\mathrm{BHJ}$ thin films offers important information to further understand the observed photovoltaic behavior of these thin film based devices. To determine the molecular packing within the neat and blend films, grazing incidence wide-angle X-ray scattering (GIWAXS) measurements were performed. The scattering signatures of neat FTAZ and PyCNTAZ films are very similar, and both exhibit clear (100) peaks at $q=$ $0.3 \AA^{-1}$ in the in-plane direction and (010) peaks located at $q=$ $1.7 \AA^{-1}$ in the out-of-plane direction (Fig. 7a and b), suggesting that the polymers have a face-on orientation with respect to the substrate. Such a face-on feature was frequently observed before in many high-performance conjugated polymers and considered to be advantageous for hole transport across the active layer. ${ }^{34}$ As shown in Fig. 7c, the neat SF-PDI 2 film does not display a (010) reflection peak; rather, it shows weak (100) diffuse rings and an amorphous halo around $q=1.3 \AA^{-1}$ without a clear orientation preference.
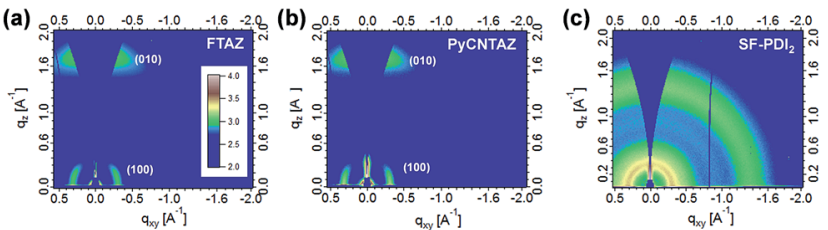

Fig. 7 GIWAXS 2D patterns of the thin films based on neat materials: (a) FTAZ; (b) PyCNTAZ; (c) SF-PDI 2 . 

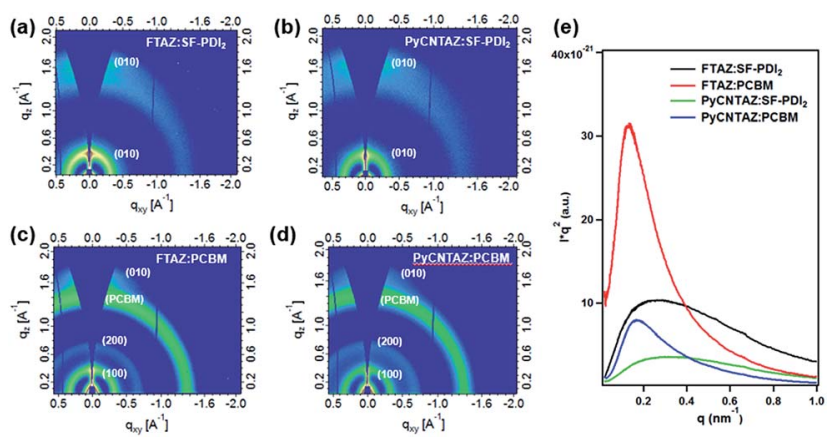

Fig. 8 GIWAXS 2D patterns of (a) FTAZ:SF-PDI ${ }_{2}$ (b) FTAZ:PCBM, (c) PyCNTAZ:SF-PDI 2 and (d) PyCNTAZ:PCBM blend films; (e) Lorentzcorrected and thickness- and contrast-normalized circular averaged $\mathrm{R}$-SoXS profiles of the blend films acquired at $283.2 \mathrm{eV}$ to optimize the polymer:acceptor contrast over the mass thickness contrast.

Shown in Fig. 8a-d are the 2D GIWAXS patterns of these polymers blended with PCBM or SF-PDI ${ }_{2}$. The SF-PDI blend films display weak (100) and (010) diffraction peaks, indicative of poor lamellar packing and $\pi-\pi$ stacking. In contrast, the PCBM-based blends exhibit more clear lamellar (100) and (200) peaks, and a (010) peak in the out-of-plane direction in addition to an isotropic ring located at $q=1.35$ $\AA^{-1}$, which is originated from pure PCBM aggregates. ${ }^{35}$ Comparison of the GIWAXS patterns between fullerene and SF$\mathrm{PDI}_{2}$-based films indicates that the PCBM-based films are more ordered, which agrees well with the higher hole/electron mobility observed for these PCBM-based devices (Table 3). A higher degree of molecular ordering in the film could afford improved charge transport and thus higher charge mobility, leading to the higher $J_{\mathrm{sc}}$ and FF values measured for the PCBMbased devices.

Resonant soft X-ray scattering (R-SoXS) was also utilized to determine the domain spacing and relative average composition variation (ACV) of the four blend films. The Lorentz corrected R-SoXS profiles acquired at a photon energy of $283.2 \mathrm{eV}$ are normalized for thickness, contrast, and absorption differences (Fig. 8e).$^{36}$ All R-SoXS profiles are dominated by a single size distribution and the relative ACV can be extracted via integration of the scattering profiles over the full $q$-range probed and normalized to the highest ACV assigned a value of 1 (Table 4). The relative ACVs are 0.83 and 1 for FTAZ:SF-PDI 2 and FTAZ:PCBM, respectively, and 0.49 and 0.56 for PyCNTAZ:SF$\mathrm{PDI}_{2}$ and PyCNTAZ:PCBM, respectively. Generally, a higher relative $\mathrm{ACV}$ (i.e., more pure domains) is important as impure

Table 4 Domain characteristics of the four blend films extracted from the R-SoXS measurements

\begin{tabular}{|c|c|c|}
\hline Blend & $\begin{array}{l}\text { Relative } \\
\text { ACV }\end{array}$ & $\begin{array}{l}\text { Long period } \\
{[\mathrm{nm}]}\end{array}$ \\
\hline FTAZ:SF-PDI ${ }_{2}$ & 0.83 & 25.6 \\
\hline FTAZ:PCBM & 1 & 49.0 \\
\hline PyCNTAZ:SF-PDI ${ }_{2}$ & 0.49 & 20.0 \\
\hline PyCNTAZ:PCBM & 0.56 & 37.7 \\
\hline
\end{tabular}

domains lead to enhanced biomolecular recombination and thus lower device FF in both fullerene and non-fullerene based OPV systems. ${ }^{37,38}$ In our case study, the highest relative ACV obtained in the FTAZ:PCBM film is quite consistent with its highest device FF up to $\sim 73 \%$. It is clear that the PCBM blends have more pure domains for both polymers, which is another factor contributing to the higher FF of the fullerene devices. The long period (domain spacing) of the SF-PDI ${ }_{2}$ films is close to the exciton diffusion length, 25.6 nm for FTAZ:SF-PDI 2 and $20.0 \mathrm{~nm}$ for PyCNTAZ:SF-PDI ${ }_{2}$. Despite having a longer long period (49.0 nm for FTAZ:PCBM and $37.7 \mathrm{~nm}$ for PyCNTAZ:PCBM), the PCBM-based devices display higher $J_{\mathrm{sc}}$ and FF values, likely due in part to the higher charge mobility of these PCBMbased systems, which allows for more efficient charge transport and extraction. As shown in the 12\%-efficiency NFA-based devices, ${ }^{38,39}$ the blend films based on IT-M are more ordered compared with the SF-PDI 2 films. We thus speculate that applying other NFAs with higher crystallinity may be the key to overcoming the current limitations and further boosting the efficiency of non-fullerene devices based on FTAZ and PyCNTAZ polymers.

\section{Conclusions}

Solar cells based on the non-fullerene acceptor SF-PDI ${ }_{2}$ have a larger $V_{\text {oc }}$ than those based on PCBM; however, they also have a lower $J_{\mathrm{sc}}$ and $\mathrm{FF}$, leading to a decreased overall power conversion efficiency. The increased $V_{\text {oc }}$ in the non-fullerene devices is explained by the higher-lying LUMO level of SF$\mathrm{PDI}_{2}$, which increases the $\mathrm{HOMO}_{\text {donor }}-\mathrm{LUMO}_{\text {acceptor }}$ gap, and more importantly, leads to a higher $E_{\mathrm{CT}}$ for these devices. In all four devices, the energy loss due to non-geminate recombination $\left(\Delta E_{\mathrm{NG}}\right)$ is very similar $(\sim 0.6 \mathrm{eV})$, yet the driving force for charge separation $\left(\Delta E_{\mathrm{CS}}\right)$ is exceptionally small $(\sim 0.03 \mathrm{eV})$ for the PyCNTAZ-based devices (with either PCBM or SF-PDI ${ }_{2}$ as the acceptor). This interesting discovery certainly warrants further investigation. For example, what structural features of molecules would lead to such a low $\Delta E_{\mathrm{CS}}$ ? On the other hand, the SF- $\mathrm{PDI}_{2}$-based devices show insufficient charge generation, transport and collection, which would explain the low $J_{\mathrm{sc}}$ of these devices. Notably, the light intensity dependence of $V_{\mathrm{oc}}$ and $J_{\mathrm{sc}}$ indicates more recombination loss channels, including trap-assisted recombination and surface recombination in the SF-PDI ${ }_{2}$-based devices, in addition to the already increased bimolecular recombination loss in these devices. All these recombination losses would account for the decreased $J_{\mathrm{sc}}$ of the SF-PDI ${ }_{2}$ based solar cells. Finally, the SF-PDI ${ }_{2}$-based devices demonstrate less pure domains, low electron mobilities, and an imbalance in the electron and hole mobilities, all of which contribute to the observed decrease in the FF.

In summary, our understanding of the causes of the decreased $J_{\mathrm{sc}}$ and $\mathrm{FF}$ generally observed in non-fullerene acceptor based OPVs can aid in the designing of new nonfullerene acceptors with improved $J_{\mathrm{sc}}$ and $\mathrm{FF}$, while maintaining the potential increase in $V_{\mathrm{oc}}$ in these non-fullerene acceptor based solar cells. 


\section{Experimental}

Synthesis

FTAZ, ${ }^{18}$ PyCNTAZ, ${ }^{19}$ and SF-PDI ${ }_{2}{ }^{17}$ were synthesized according to a literature procedure. The purity of all synthesized molecular materials was confirmed by NMR analysis.

\section{Device fabrication}

Solar cells were fabricated on glass substrates with patterned indium doped tin oxide (ITO). The ITO substrates were sonicated in deionized water, acetone, and isopropyl alcohol for fifteen minutes each, followed by UV-ozone treatment for 15 minutes. For FTAZ devices, PEDOT:PSS (Clevios PH500 from Heraeus) was spin cast onto the cleaned ITO at $4000 \mathrm{rpm}$ for $60 \mathrm{~s}$, and then baked at $130{ }^{\circ} \mathrm{C}$ for fifteen minutes in air. The substrates were then transferred into a nitrogen filled glovebox. For PyCNTAZ devices, CuSCN was dissolved in dipropylsulfide $\left(20 \mathrm{mg} \mathrm{mL}^{-1}\right)$ and stirred for $24 \mathrm{~h}$, after which the saturated solution was filtered with a $0.2 \mu \mathrm{m}$ poly(tetrafluoroethylene) (PTFE) filter. CuSCN was spin cast onto the cleaned ITO substrate at $1000 \mathrm{rpm}$ for $60 \mathrm{~s}$, and then baked at $80^{\circ} \mathrm{C}$ for $15 \mathrm{~min}$ in a glovebox under a nitrogen atmosphere. Donor : acceptor blend solutions were prepared (D : A = 1:2, $7 \mathrm{mg} \mathrm{mL} \mathrm{m}^{-1}$ polymer for all four solutions) in 1,2,4-trichlorobenzene and stirred at $130{ }^{\circ} \mathrm{C}$ for $5 \mathrm{~h}$. The solutions were spin cast onto the PEDOT:PSS or CuSCN films for $60 \mathrm{~s}$ at an appropriate speed and then dried under vacuum to yield $\sim 150 \mathrm{~nm}$ films. The devices were finished by evaporation of $30 \mathrm{~nm}$ of calcium and $70 \mathrm{~nm}$ of aluminum as the cathode and tested under AM 1.5G irradiation calibrated with an NREL certified standard silicon solar cell. Current density-voltage curves were measured via a Keithley 2400 digital source meter.

\section{SCLC measurements}

Electron and hole mobilities were measured via the spacecharge limited current (SCLC) method. Electron-only devices were fabricated with the configuration ITO/PEI/donor:acceptor/ $\mathrm{Ca} / \mathrm{Al}$, where PEI is polyethyleneimine, used for reducing the work function of ITO. ${ }^{40}$ Hole-only devices were fabricated with the configuration ITO/HTL/donor:acceptor/ $\mathrm{MoO}_{3} / \mathrm{Al}$, where the HTL was PEDOT:PSS for FTAZ-based devices and CuSCN for PyCNTAZ-based devices. The dark current densities were measured with an applied voltage from 0 to $6 \mathrm{~V}$ using a Keithley 2400 digital source meter. The applied voltage was corrected from the voltage drop due to series and contact resistance. The Mott-Gurneys law was utilized to extract the mobility values:

$$
J=\frac{9}{8} \varepsilon_{\mathrm{r}} \varepsilon_{0} \mu_{\mathrm{h}} \frac{V^{2}}{L^{3}}
$$

where $\varepsilon_{\mathrm{r}}$ is the dielectric constant of the polymer, $\varepsilon_{0}$ is the permittivity of free space, $\mu_{\mathrm{h}}$ is the hole mobility, $V$ is the voltage drop across the device, and $L$ is the thickness of the active layer.

\section{Long wavelength EQE measurements}

Devices for long wavelength EQE measurements were spin cast at the University of North Carolina Chapel Hill, and the top contacts were evaporated at North Carolina State University.
Active layer thicknesses were $\sim 150 \mathrm{~nm}$ for both FTAZ devices and $\sim 120 \mathrm{~nm}$ for PyCNTAZ devices. Long wavelength EQE measurements were conducted using an in-house setup consisting of a xenon DC arc lamp, an ORIEL 74125 monochromator, a Keithley 428 current amplifier, an SR 540 chopper system and a Stanford Research Systems SR830 DSP lock-in amplifier. For the calibration of the spectrum, a Si and a Ge photodiode purchased from Newport Corporation were used as necessary. $700 \mathrm{~nm}$ and $1000 \mathrm{~nm}$ long-pass filters were used in order to isolate the desired part of the spectrum for the monitoring of the sub-bandgap response.

\section{Morphology}

GIWAXS, R-SoXS and NEXAFS reference spectra measurements were respectively performed at the beamline 7.3.3 ${ }^{\mathbf{4 1}}$, beamline 11.0.1.2, ${ }^{42}$ and beamline 5.3.2.2, ${ }^{43}$ Advanced Light Source (ALS), Lawrence Berkeley National Laboratory, following the previously established protocols. GIWAXS data were acquired just above the critical angle $\left(0.13^{\circ}\right)$ of the films with a hard X-ray energy of $10 \mathrm{keV}$, and silver behenate (AgB) was used for geometry calibration. R-SoXS was performed in a transmission geometry with linearly polarized photons under high vacuum (1 $\times 10^{-7}$ Torr) and a cooled $\left(-45^{\circ} \mathrm{C}\right) \mathrm{CCD}$ (Princeton PI-MTE, 2048 pixels $\times 2048$ pixels) was used to capture the soft X-ray scattering 2D maps and a PS300 was used for geometry calibration. The raw 2D X-ray data were processed with a modified version of NIKA into $1 \mathrm{D}$ scattering profiles $I(q){ }^{44}$

\section{Acknowledgements}

NB, QZ, and WY were supported by the Office of Naval Research (Grant No. N000141410221), and a NSF grant (DMR-1507249). WY and LY were supported by a NSF grant (ECCS-1344745). HY and JZ thank the Hong Kong Innovation and Technology Commission for the support through ITC-CNERC14SC01. GIWAXS/R-SoXS data acquisition and analysis by LY and HA were supported by the ONR grant N00141512322. IC and FS acknowledge the support from the Office of Naval Research (N00014-14-1-0173). X-ray data were acquired at beamlines 11.0.1.2, 5.3.2.2, and 7.3.3 at the ALS in the Berkeley National Lab, which was supported by the U.S. Department of Energy (DE-AC02-05CH11231). J.-H. Kim is acknowledged for assisting with part of the X-ray data acquisition. We also thank Wesley Swords and Professor Gerald Meyer for assistance with PL measurements.

\section{Notes and references}

1 Y. He and Y. Li, Phys. Chem. Chem. Phys., 2011, 13(6), 19701983.

2 S. Li, Z. Zhang, M. Shi, C.-Z. Li and H. Chen, Phys. Chem. Chem. Phys., 2017, 19, 3440-3458.

3 Y. Lin, F. Zhao, Q. He, L. Huo, Y. Wu, T. C. Parker, W. Ma, Y. Sun, C. Wang, D. Zhu, A. J. Heeger, S. R. Marder and X. Zhan, J. Am. Chem. Soc., 2016, 138(4), 4955-4961. 
4 T. Liu, D. Meng, Y. Cai, X. Sun, Y. Li, L. Huo, F. Liu, Z. Wang, T. P. Russell and Y. Sun, Adv. Sci., 2016, 3, 1600117.

5 H. Yao, Y. Chen, Y. Qin, R. Yu, Y. Cui, B. Yang, S. Li, K. Zhang and J. Hou, Adv. Mater., 2016, 1-5.

6 L. Gao, Z. Zhang, H. Bin, L. Xue, Y. Yang, C. Wang, F. Liu, T. P. Russell and Y. Li, Adv. Mater., 2016, 1-8.

7 W. Zhao, D. Qian, S. Zhang, S. Li, O. Inganäs, F. Gao and J. Hou, Adv. Mater., 2016, 28(23), 4734-4739.

8 S. Li, L. Ye, W. Zhao, S. Zhang, S. Mukherjee, H. Ade and J. Hou, Adv. Mater., 2016, 28, 9423-9429.

9 Z. He, B. Xiao, F. Liu, H. Wu, Y. Yang, S. Xiao, C. Wang, T. P. Russell and Y. Cao, Nat. Photonics, 2015, 9(3), 174-179.

10 V. Vohra, K. Kawashima, T. Kakara, T. Koganezawa, I. Osaka,

K. Takimiya and H. Murata, Nat. Photonics, 2015, 9, 403-409.

11 J. Zhao, Y. Li, G. Yang, K. Jiang, H. Lin, H. Ade, W. Ma and H. Yan, Nat. Energy, 2016, 1(2), 15027.

12 Y. Lin and X. Zhan, Mater. Horiz., 2014, 1(5), 470-488.

13 G. Lakhwani, A. Rao and R. H. Friend, Annu. Rev. Phys. Chem., 2014, 65(1), 557-581.

14 W. Li, S. Albrecht, L. Yang, S. Roland, J. R. Tumbleston, T. McAfee, L. Yan, M. A. Kelly, H. Ade, D. Neher and W. You, J. Am. Chem. Soc., 2014, 136(44), 15566-15576.

15 Y. Lin, P. Cheng, Y. Li and X. Zhan, Chem. Commun., 2012, 48(39), 4773.

16 D. Meng, D. Sun, C. Zhong, T. Liu, B. Fan, L. Huo, Y. Li, W. Jiang, H. Choi, T. Kim, J. Y. Kim, Y. Sun, Z. Wang and A. J. Heeger, J. Am. Chem. Soc., 2016, 138(1), 375-380.

17 J. Zhao, Y. Li, H. Lin, Y. Liu, K. Jiang, C. Mu, T. Ma, J. Y. Lin Lai, H. Hu, D. Yu and H. Yan, Energy Environ. Sci., 2015, 8(2), 520-525.

18 S. C. Price, A. C. Stuart, L. G. Yang, H. X. Zhou and W. You, J. Am. Chem. Soc., 2011, 133, 4625-4631.

19 W. Li, L. Yan, H. Zhou and W. You, Chem. Mater., 2015, 27(18), 6470-6476.

20 W. Li, L. Yang, J. R. Tumbleston, L. Yan, H. Ade and W. You, Adv. Mater., 2014, 26(26), 4456-4462.

21 E. T. Hoke, K. Vandewal, J. A. Bartelt, W. R. Mateker, J. D. Douglas, R. Noriega, K. R. Graham, J. M. J. Fréchet, A. Salleo and M. D. Mcgehee, Adv. Energy Mater., 2013, 3(2), 220-230.

22 K. Vandewal, K. Tvingstedt, A. Gadisa, O. Inganäs and J. V. Manca, Nat. Mater., 2009, 8, 904-909.

23 K. Vandewal, K. Tvingstedt, A. Gadisa, O. Inganäs and J. V. Manca, Phys. Rev. B: Condens. Matter Mater. Phys., 2010, 81(12), 125204.

24 M. A. Faist, T. Kirchartz, W. Gong, R. S. Ashraf, I. Mcculloch, J. C. De Mello, N. J. Ekins-Daukes, D. D. C. Bradley and J. Nelson, J. Am. Chem. Soc., 2011, 134(1), 685-692.

25 J. Liu, S. Chen, D. Qian, B. Gautam, G. Yang, J. Zhao, J. Bergqvist, F. Zhang, W. Ma, H. Ade, O. Inganäs,
K. Gundogdu, F. Gao and H. Yan, Nat. Energy, 2016, 1(9), 16089.

26 A. K. K. Kyaw, D. H. Wang, V. Gupta, W. L. Leong, L. Ke, G. C. Bazan and A. J. Heeger, ACS Nano, 2013, (5), 4569-4577.

27 L. Lu, T. Zheng, T. Xu, D. Zhao and L. Yu, Chem. Mater., 2015, 27(2), 537-543.

28 S. R. Cowan, A. Roy and A. J. Heeger, Phys. Rev. B: Condens. Matter Mater. Phys., 2010, 82(24), 245207.

29 V. V. Brus, Org. Electron., 2016, 29, 1-6.

30 S. Solak, P. W. M. Blom and G. A. H. Wetzelaer, Appl. Phys. Lett., 2016, 109(5), 053302.

31 P. W. M. Blom, V. D. Mihailetchi, L. J. A. Koster and D. E. Markov, Adv. Mater., 2007, 19(12), 1551-1566.

32 L. Yang, J. Tumbleston, H. Zhou, H. Ade and W. You, Energy Environ. Sci., 2012, 316-326.

33 C. M. Proctor, J. A. Love and T.-Q. Nguyen, Adv. Mater., 2014, 26(34), 5957-5961.

34 P. M. Beaujuge and J. M. J. Fréchet, J. Am. Chem. Soc., 2011, 133(50), 20009-20029.

35 J. A. Bartelt, Z. M. Beiley, E. T. Hoke, W. R. Mateker, J. D. Douglas, B. A. Collins, J. R. Tumbleston, K. R. Graham, A. Amassian, H. Ade, J. M. J. Fréchet, M. F. Toney and M. D. McGehee, Adv. Energy Mater., 2013, 3(3), 364-374.

36 J. H. Carpenter, A. Hunt and H. Ade, J. Electron Spectrosc. Relat. Phenom., 2015, 200, 2-14.

37 S. Mukherjee, X. Jiao and H. Ade, Adv. Energy Mater., 2016, 6(18), 1600699.

38 L. Ye, W. Zhao, S. Li, S. Mukherjee, J. H. Carpenter, O. Awartani, X. Jiao, J. Hou and H. Ade, Adv. Energy Mater., 2016, 1602000.

39 S. Li, L. Ye, W. Zhao, S. Zhang, S. Mukherjee, H. Ade and J. Hou, Adv. Mater., 2016, 28, 9423-9429.

40 Y. Zhou, C. Fuentes-Hernandez, J. Shim, J. Meyer, A. J. Giordano, H. Li, P. Winget, T. Papadopoulos, H. Cheun, J. Kim, M. Fenoll, A. Dindar, W. Haske, E. Najafabadi, T. M. Khan, H. Sojoudi, S. Barlow, S. Graham, J.-L. Bredas, S. R. Marder, A. Kahn and B. Kippelen, Science, 2012, 336(6079), 327-332.

41 A. Hexemer, W. Bras, J. Gossinger, E. Schaible, E. Gann, R. Kirian, A. MacDowell, M. Church, B. Rude and H. Padmore, J. Phys.: Conf. Ser., 2010, 247(1), 12007.

42 E. Gann, A. T. Young, B. A. Collins, H. Yan, J. Nasiatka, H. A. Padmore, H. Ade, A. Hexemer and C. Wang, Rev. Sci. Instrum., 2012, 83(4), 45110.

43 A. L. D. Kilcoyne, T. Tyliszczak, W. F. Steele, S. Fakra, P. Hitchcock, K. Franck, E. Anderson, B. Harteneck, E. G. Rightor, G. E. Mitchell, A. P. Hitchcock, L. Yang, T. Warwick and H. Ade, J. Synchrotron Radiat., 2003, 10, 125-136.

44 J. Ilavsky, J. Appl. Crystallogr., 2012, 45(2), 324-328. 